\title{
Long-baseline optical fiber interferometer instruments and science
}

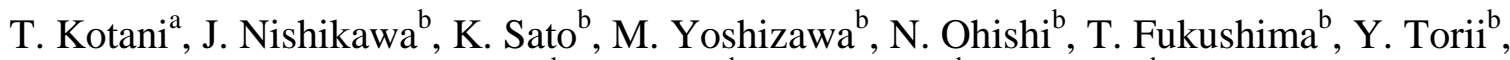 \\ K. Matsuda ${ }^{\mathrm{b}}$, K. Kubo ${ }^{\mathrm{b}}$, H. Iwashita ${ }^{\mathrm{b}}$, S. Suzuki ${ }^{\mathrm{b}}$ \\ ${ }^{a}$ Department of Astronomy, Graduate School of Science, the University of Tokyo, Japan \\ ${ }^{b}$ MIRA project, National Astronomical Observatory of Japan
}

\begin{abstract}
Developments of fiber linked optical interferometer are reported. This interferometer is a part of MIRA-I.2 interferometer (Mitaka InfraRed and optical Array). MIRA-I.2 is an optical interferometer with a 30 meters long baseline. It consists of two $30 \mathrm{~cm}$ siderostats, tip-tilt mirrors, vacuum pipes delay lines and detectors. We plan to use two 60 meters long polarization-maintaining fibers for arms of the interferometer, instead of vacuum pipes. The developments include dispersion and polarization compensation of fiber and fiber injection module. In laboratory experiments, dispersion compensation succeeded. The fringe visibility was 0.93 for wide-band, where the central wavelength of light was $700 \mathrm{~nm}$, and bandwidth was $200 \mathrm{~nm}$, while 0.95 with a He-Ne laser. We used BK7 glass wedge for dispersion compensation. About fiber injection module, basic optical design has completed. The results of our fiber interferometer could contribute to OHANA (Optical Hawaiian Array for Nanoradian Astronomy) project. We present new science targets, white dwarves and T Tauri stars, and an 800m delayline concept in CFHT for the project.
\end{abstract}

Keywords: Fiber, optical interferometry, OHANA, science, delay line

\section{INTRODUCTION}

Single-mode fibers have been used for spatial filtering which reduce the effects of wavefront errors by the atmospheric turbulence and incomplete beam-combiner optics ${ }^{1}$. On the other hand, single-mode fibers are good devices of coherent beam transport for long baseline optical interferometers. Conventional stellar interferometers using vacuum pipes or air tunnels for beam transport have several problems: misalignment of optical axis, high cost for construction of vacuum pipes, difficulties for construction of long vacuum pipe path on complex landscape, and so on. The single-mode fiber is the most suitable device to solve these problems, particularly in case of OHANA project which combine big telescopes on the top of Mauna Kea ${ }^{2,3,4}$. However, to preserve coherency of the light is difficult in very long distance beam transport with fibers, which have some effects reducing coherency of the light. The first is that optical fibers display dispersion significantly. If there are differences of fiber length between two beam-transport fibers in an interferometer, the fringe phase varies non-linearly in wavelength. Hence a resultant wide-band fringe will have low visibility by the sum of not co-phased fringes. The second is that the polarization state of the light ejected from the fibers varies due to the birefringence by bending and twist of fibers which also reduces the fringe contrast. Previously several groups have investigated fibers for coherent beam transport ${ }^{1,5}$. A wide-bandwidth light $(650-850 \mathrm{~nm})$ experiment using three $25 \mathrm{~m}$ long polarization maintaining fibers were reported obtaining high contrast fringes ${ }^{6}$. This was archived by controlling optical path stability and correcting dispersion and polarization differences. In case of 500m-long highly birefringent fibers, moderate fringe contrast was observed for wide-width $(140 \mathrm{~nm})$ light at $1270 \mathrm{~nm}$ wavelength ${ }^{7}$.

MIRA $^{8,9,10}$ (Mitaka optical and InfraRed Array) group has proposed fiber connected stellar interferometer where Subaru telescope is combined with other big telescopes on the top of Mauna $\mathrm{Kea}^{3,4}$. The project, called MIRA-SG, has merged into OHANA from December 2000. We have been making basic experiments of fiber interferometer for these projects. Sato et al. reported high contrast fringes using $10 \mathrm{~m}$ standard fibers ${ }^{11}$. The visibility was 0.7 for wide-bandwidth light and 0.75 for He-Ne laser without maintenance of polarization state. It was archived with dispersion compensation with BK7 wedge glass. In the present paper, we report the experimental results of higher fringe contrast with two types of longer fibers, and several unique ideas for OHANA. In section 2, we describe interferometric experiments with two $60 \mathrm{~m}$-long highly birefringent fibers. We also describe achromatic polarization compensation method using 100m-long standard fibers. In section 3, a new method to measure the fiber length, particularly lied between telescopes is shown. In section 4, we suggest new scientific targets for OHANA, T Tauri stars and white dwarves. A new 800m delayline concept for OHANA is also described. 


\section{THE INTERFEROMETRIC EXPERIMENTS}

\subsection{GENERAL DESCRIPTION OF EXPERIMENT}

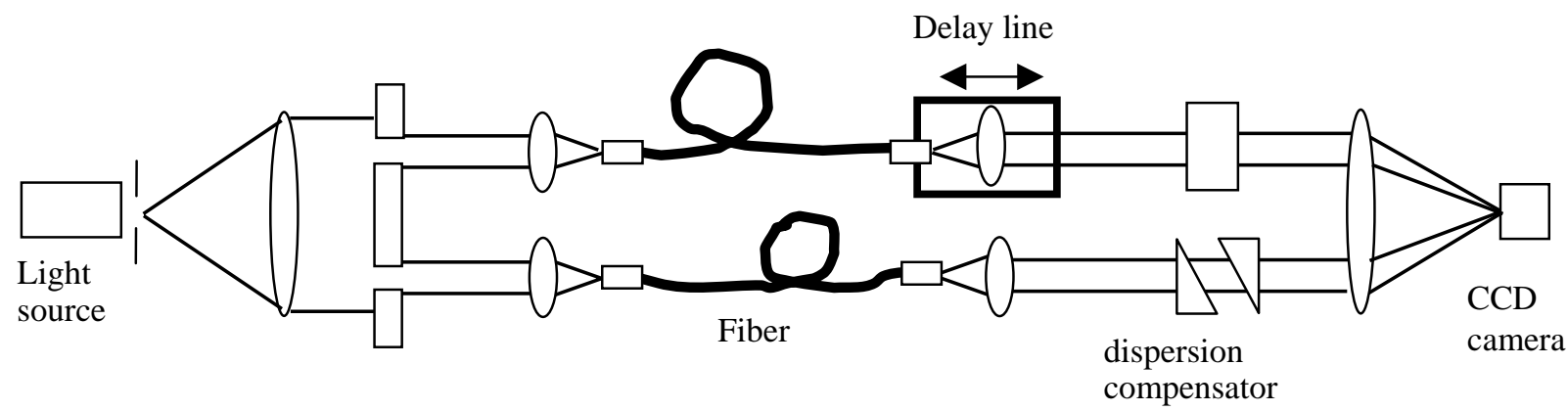

Fig. 1. Overall layout of the interferometric experiment

The experimental set up is shown in the Fig. 1. This is a Fizeau type interferometer where each interferometer arm was replaced with single-mode fibers. We used two types of fibers. One is 60m-long highly birefringent fiber (Fujikura SMC-63P), maintaining polarization state. Another is 100m-long standard fiber (SpecTran BC03817). The cut-off wavelength for each fiber is $600 \mathrm{~nm}$. In order to equalize the length of the fibers, we used a laser range meter (MM30R, Sokkia Co. Ltd), and cut the fibers with a length difference of a few mm, enabling easy dispersion compensation with a glass plate ${ }^{11}$. The method of fiber length equalization is described in section 3 in detail.

Two light sources, a He-Ne laser and a wide-bandwidth light $(600 \mathrm{~nm}-760 \mathrm{~nm})$, are used for the experiment. The light goes through a pinhole and is collimated by a lens. The light is stopped by a two-hole diaphragm and focused onto two fiber ends by lens. In the case of using polarization-maintaining fiber, the ejected light is exactly linearly polarized with a polarizer, which polarization angle is the same with the polarization maintaining fiber. A random polarization light is used for standard fiber. In the output configuration, the beams were focused and recombined with a lens and observed by a CCD camera. The interference fringes were observed in a diffraction image core. We tuned the optical path by moving one of the collimator lens and fiber ends on a DC servo translation stage. A BK7 wedge glass achieved the compensation of chromatic dispersion.

\subsection{RESULT OF EXPERIMENT: POLARIZATION MAINTAINING FIBERS}

When the dispersion is compensated with BK7 wedge glass, the fringe visibility improved from $45 \%$ to $93 \%$ for the wide-band source. The input light is linearly polarized, and polarization state is maintained for the output light by the fiber and polarizer. In this way, dispersion and polarization problems were solved.

\subsection{STANDARD FIBERS}

In the case of 100m-long standard fibers, both dispersion and polarization compensation are required. Bending of a fiber introduces birefringence, which corresponds to a waveplate whose retardation depends on wavelength. Consequently the polarization state of output light from different fiber does not match each other. It results in a degradation of coherency of the light ${ }^{5}$.

When the fiber is short, the amount of bending is small, so that the wavelength dependence of polarization is small. Hence it is easy to match polarization state for wide-band by the change of bending ${ }^{1}$. When the fiber is long, there are 
many bendings and twists, and wavelength dependence of polarization becomes large. Fig. 2. (a) shows samples of polarization state of the output light. In this case, matching polarization state for wide wavelength band is very difficult because the wavelength dependence of the polarization state remains. Actually, in a measurement with the $100 \mathrm{~m}$ standard fibers, without any adjustment of polarization, the fringe contrast for wide-band source was $45 \%$ and for HeNe laser $70 \%$. When we match the polarization state at He-Ne laser wavelength by using several fiber loops, fringe visibility was 0.5 for the wide-band light, and 0.95 for He-Ne laser. This is not adequate because real observation needs the wide-band light. Therefore, we need to remove wavelength dependence of the polarization state.

To simplify the problem, let us consider two fiber loops whose retardation property is the same but fast axis is perpendicular for each other. The wavelength-dependence of retardation caused by the first bending is canceled by that of the second bending for all wavelengths, and the polarization state is restored to that of the input light. A composition of bending and twist in the real fiber case can be modeled as a combination of retarders and rotators. The retardation property of fiber is derived from wavelength dependence of the polarization state. Thus we can restore polarization state by using appropriate fiber loops.

\subsection{SIMULATION RESULTS OF WIDE-BAND POLARIZATION COMPENSATORS FOR STANDARD FIBERS}

We have designed a numerical model of a wide-band polarization compensator consisting of fiber loops, and then applied it to the measured data of the output polarization state at the ejected light from the $100 \mathrm{~m}$ standard fibers. Fig. 2 (a) indicates measured wavelength-dependences of polarization state for two fibers. Fig. 2 (b) shows compensated polarization state. The polarization states of the two fibers were restored to almost linear polarization for wide wavelength band. When these two light are interfered, the fringe visibility becomes $88 \%$ for the wide-band light source and $95 \%$ for He-Ne laser wavelength. This result indicates that the fringe visibility could be significantly improved, when wide-band polarization compensators are used.

(a)

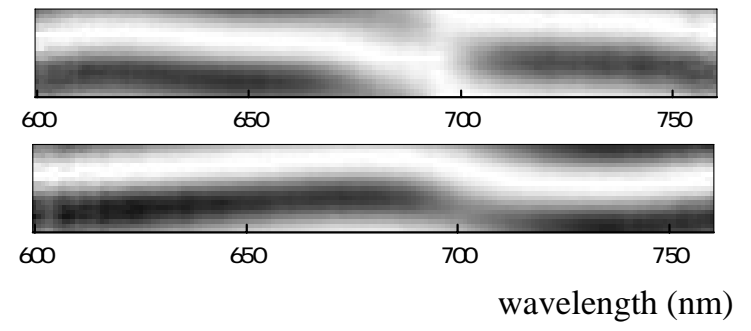

(b)

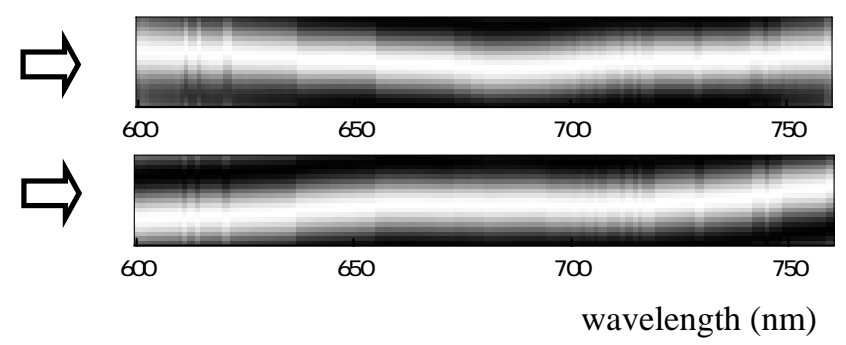

Fig. 2. Polarization states of light ejected from $100 \mathrm{~m}$ standard fibers. Ordinate is the angle of the linear analyzer. Abscissa is the wavelength. The gray scale shows intensity of the light through the analyzer. (a): Measured polarization states. (b) Polarization states after wide-band polarization compensation by numerical simulation.

\section{EQUALIZATION OF FIBER LENGTHS FOR ZERO DISPERSION WIDE-BAND OBSERVATIONS}

Equalizing fiber lengths is essential to make wide-band observations in optical fiber interferometer. When an optical path length difference between the two arms of the interferometer in dispersion material is zero, the light in all wavelength produce constructive fringes at the same time at zero delay condition yielding less visibility degradations for wide-band fringes. In the fiber case both material and waveguide dispersion affect the optical path length for wideband light. How to reduce and compensate the differences of fiber length at 10um level is described 
Firstly we show in the laboratory case as (A).

(A-1) To measure the fiber length with a precision of a few $\mathrm{mm}$ for a $100 \mathrm{~m}$ fiber by using a laser range meter [see section 2]. The differences between optical path lengths of the fibers can be as accurate as $1 \mathrm{~mm}$ although the absolute lengths have larger errors caused by the fluctuation of refractive index of the long fibers. The fibers should be cut at the same length as precise as possible.

(A-2) To measure a delay line position of white light fringes at a fiber interferometer. Differences of the delay line position when exchanging the fibers in the two arms of the interferometer indicate the differences of the fiber optical path length. This method is more accurate than the measurements with the laser range meter, but the fiber length should be almost the same length.

(A-3) To compensate the fiber optical path length by using wedge glass plate looking at the wide-band fringes. It is to search the maximum visibility by changing the thickness of the glass plate. Channeled spectrum indicating fringe patterns for each wavelength help us to find the appropriate thickness of the glass compensator. For the best compensator thickness and the delay line position, the fringes show constructive condition at the same time making the channel straight, which indicates the lengths of the dispersion materials coincide as well as the length of the air.

Next we discuss how to equalize the fiber length when the fibers are laid from telescopes to a beam-combining room as case(B). The fiber optical length would change by bends, tension, and temperature differences.

(B-1) To measure the fiber lengths like (A-1) but here two fibers should be connected at telescope end enabling to use them as one fiber, so that two ends are accessible at the beam-combining room. We are confirming the measurements of connected fiber lengths with the laser range meter. It was difficult to use the laser range meter inserting the light at an end of the fiber and detecting the light at the same end reflected at the other side, because many strong ghost light disturbed the measurements. When there are three fibers from the room to a telescope, three measured lengths of three combinations of fibers give us each length of fibers by solving simultaneous equations. When the fiber lengths are largely different, they must be cut again in the same length, or a short fiber should be connected to an end by a fiber coupler.

(B-2) To measure a delay line position for white light fringes exchanging the fibers at a fiber interferometer like (A-2). In this case it may be possible to use the wide-band light inserting at an end of the fiber and detecting the light at the same end by returning the light at the other side. If impossible, connected fibers and solving equations as (B-1) should be adopted.

(B-3) To compensate the fiber optical path length by using wedge glass plate looking at the wide-band fringes as (A-3) with the successful setup of (B-2).

(B-4) The fiber length could be changed by temperature drift or bending of the fiber according to telescope pointing. In order to compensate the fiber length variation, a fringe tracking with an artificial light is promising. Fringe tracking in a different wavelength in one fiber is possible ${ }^{12}$. When more than three fibers from the beam-combining room to a telescope are there, one fiber can be used for observations and other fibers are for fiber length tracking. This method would be stable if the fibers are involved in a cladding protection cable.

\section{OHANA}

In this section, we propose other possible science cases with OHANA, T Tauri stars and white dwarves than discussed previously in the community ${ }^{4}$. A new $800 \mathrm{~m}$ delay line concept for OHANA is also described here.

\subsection{OHANA SCIENCE: T TAURI STARS}

$\mathrm{T}$ Tauri stars are in the stage evolving in accordance with gravitational contraction process after their birth with brightening under equilibrium towards zero-age main sequence. One unknown problem is that when surrounding gas stop collapsing by a wind, which is relating to the stellar masses. Another unverified theme is their structure evolutions. 
To determine the luminosity, effective temperature (or radius), mass and age of $\mathrm{T}$ Tau stars under contracting evolution, as well as stable main sequence stars, provides strong constraints for the present theoretical understanding of physics for the interior. Interferometric measurements of diameter of the stars are able to make the stellar parameters accurate when their positions in HR diagram derived from photometry and spectroscopy have insufficient accuracy. In addition to the measurements, stellar mass determinations from binary orbit observations (of interferometry), combined with theoretical iso-clone evolution tracks, are one of the most effective way for the study of evolution physics of $\mathrm{T}$ Tau stars.

A diameter of $\mathrm{T}$ Tau star is typically ten solar-diameter which corresponds to 0.7 mas for Taurus region 140pc distance and $0.1 \mathrm{mas}$ for $1 \mathrm{kpc}$, although the diameter has a variety as a function of its mass and age. The first zero drop of the visibility function for a uniform stellar disk is 0.39 mas for J-band, 0.52mas for H-band and 0.69mas for K-band with an 800m longest baseline of OHANA. In order to determine the diameter precisely, an agreement between uniform disk diameters from two (or three) wavelength bands rejecting emission lines by filters from $\mathbf{J}$ and $\mathrm{H}$ (and $\mathrm{K}$ ), is required ensuring no effect from surrounding disk.

The targets must be in the last stage of pre-main sequence evolution from weak-line $\mathrm{T}$ Tau stars to zero-age main sequence. When only K-band diameter shows a larger diameter than the one from J- and H-band, it may be in the stage where the major parts of disk gaseous have been just blown away.

Duplicity of T Tau stars, orbital motion of binary system, must be surveyed by not only interfereometric observations but also by photometric and spectroscopic instruments. A 1 AU orbit at $140 \mathrm{pc}$ can be seen as a $7 \mathrm{mas}$ ellipsoid, for $1 \mathrm{kpc}$ as 1 mas. OHANA has a suitable resolution for such binary orbit observations.

\subsection{OHANA SCIENCE: WHITE DWARVES}

White dwarves are the last evolution stage of medium mass stars. When such a star exhausted all their nuclear fuel, only the hot density core remains, a white dwarf. White dwarf consists of degenerate matter supported by the degeneracy pressure of the electron gas. We can infer following things from detailed observations of white dwarves combining with the theory; the history of star formation in the Galaxy, stellar evolutionary timescales, the mass loss processes that determine the initial mass to final mass relation, and cooling.

One of important parameters of a white dwarf is mass. There are two ways to determine the white dwarf mass. One is, to derive it indirectly from measurements of radius and surface gravity. The radius is from measurements of effective temperature, bolometric magnitude and parallax. But in this method, accuracy is low due to the error of parallax and surface gravity. The other accurate method depends on orbital solutions for binary stars. $10 \%$ of white dwarves are known as binary systems and there might be many undetected binaries including white dwarves. There is a possibility that masses can be determined accurately from interferometric measurements of close white dwarf binaries.

The longest baseline $800 \mathrm{~m}$ of OHANA has a resolution of 0.3 mas in J-band. As an example, a 0.1AU separation WD binary at $30 \mathrm{pc}$ which absolute magnitude is $10 \mathrm{mag}$ will be observed as a 3 mas separation double star of $12.4 \mathrm{mag}$. There may be a possibility OHANA discovers many white dwarf binaries. The radii of white dwarves are very small. So the largest apparent diameter is expected to be 0.07 mas of Sirius B. With the highest resolution of OHANA, a few WD's (Sirius B, Farmanen star, Proxyon B, 40 Eri B) will be able to observe their apparent diameters at higher fringe visibilities than 0.94 at J-band, independent of photometric and spectroscopic determinations of bolometric flux and effective temperature. If a wavelength 0.6 micron is available, the visibility of Sirius B decrease to 0.77 increasing the accuracy of diameter measurements. 


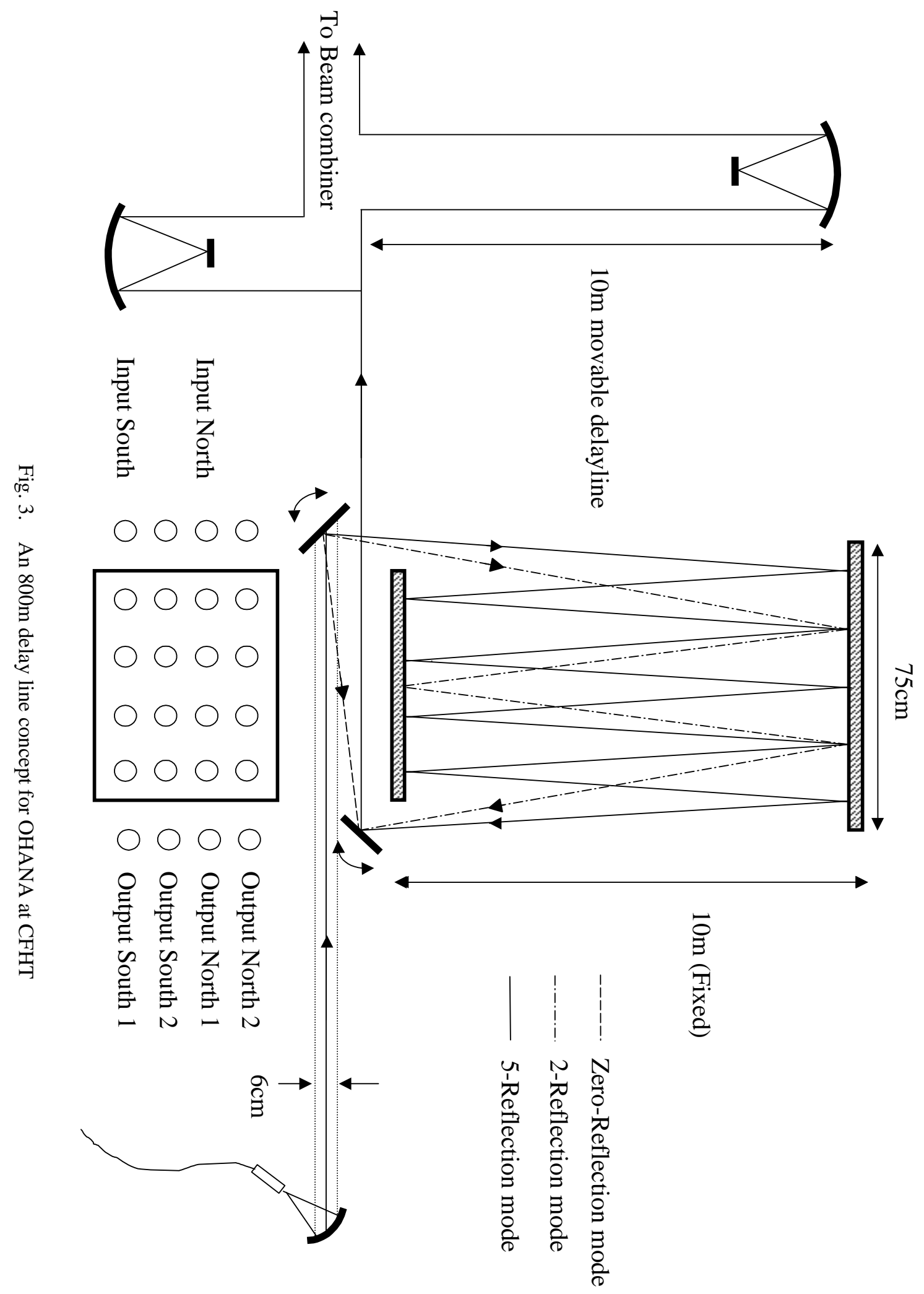




\subsection{AN 800M DELAY LINE CONCEPT FOR OHANA AT CFHT}

OHANA ${ }^{13}$ has the maximum baseline of $800 \mathrm{~m}$ which requires a compensation of a few hundred meters delay under no exclusive delay line building condition. In the Phase II of the project, a delay line system of +-40m for the early stages is considered to be put in the coude room of $\mathrm{CFHT}^{14}$, because Keck delay line system will not be always available for various baselines of OHANA. It is desirable to construct a delay line system for hundreds of meters delay in the CFHT room for the late stages ${ }^{15,16}$.

In Fig. 3 we show a concept of long delay line compensating +-400m delay. We set a collimated beam diameter $6 \mathrm{~cm}$ (at least) to propagate it as far as $400 \mathrm{~m}$. The beam is injected into a space where two flat mirrors are placed in parallel separated by $10 \mathrm{~m}$. It can be returned at the exit position after reflecting one to ten times go and back between the two mirrors yielding the optical path length from $20 \mathrm{~m}$ to $200 \mathrm{~m}$. It can be increased to $400 \mathrm{~m}$ when the beam goes through the space again. Almost $0 \mathrm{~m}$ path can be selected by not entering into the parallel mirror space. The optical path length control is done by controlling the entrance mirror(s) and the exit mirror(s). The parallel mirror long delay line provides discrete path lengths. A continuously movable fast delay line which is longer than the discrete length is required to track the star delay without delay gaps. A PZT fine delay line can be put at the focus on the fast delay line cat's eye. After passing trough the discrete long delay line and the continuous fast delay line, the beam is lead to the beam combiner. The $40 \mathrm{~m}$ delay line system ${ }^{14}$ may be adopted instead of the fast delay line in our concept. In order to compensate optical path length in the air caused by geometric height differences of the telescopes or star direction, compensation with wedge glass or evacuation of the parallel mirror long delay line may be required.

\section{CONCLUSION}

In this paper, we have presented several experimental results concerning fiber interferometer. In the case of $60 \mathrm{~m}$ polarization maintaining fibers, the wide-band fringe visibility was significantly improved, from 0.5 to 0.93 , if BK7 glass dispersion compensator was used, while the fringe visibility was 0.95 for $\mathrm{He}-\mathrm{Ne}$ laser. In the case of $100 \mathrm{~m}$ standard fiber, the visibility was $50 \%$ for wide-band source, $95 \%$ for He-Ne laser, when narrow-band polarization compensation at He-Ne laser wavelength was made. We proposed wide-band polarization compensation for higher fringe contrast. This method is demonstrated with numerical simulation, yielding the visibility was $88 \%$ for wide-band source, 95\% for He-Ne laser source. We will apply these experimental results to real interferometer like MIRA-I.2. We are planning to test beam transport by using fiber instead of vacuum pipes of MIRA-I.2. In addition, we proposed new science target and delay line concepts for OHANA, and new method to equalize the fiber length when the fibers are laid from telescopes to a beam-combining room.

\section{REFERENCES}

1. V. Coudé du Foresto and S. Ridgway, "FLOUR: A stellar interferometer using single-mode infrared fibers", ESO Proc. 39, pp. 731-740, 1992.

2. J-M. Mariotti, V. Coudé du Forest, G. Perrin, P. Zhao and P. Lena, "Interferometric connection of large groundbased telescopes", Astron. Astrophys. Suppl. Ser. 116, pp. 381-393, 1996.

3. J. Nishikawa, K. Sato, T. Fukushima, M.Yoshizawa, Y. Machida, Y. Honnma, "MIRA-II, MIRA-III, and MIRASG project: the future plan of long-baseline optical/IR interferometrer in Japan", Proc. of SPIE, 3350, pp. 184-191, 1998.

4. O. Lai, S. T. Ridgway, X. Delfosse, C. Dougados, V. Coudé du Foresto, O. Guyon, R. Lachaume, E. Magnier, F. Malbet, F. Menard, D. Mourard, G. Perrin, H. Sol, S. Warren, J. Woillez, "OHANA: representative science objectives", Proc. SPIE, 4838, submitted, 2002

5. S. Shaklan and R. Roddier, "Single-mode fiber optics in a long-baseline interferometer", Appl. Opt, 26, 11, pp. 2159-2163, 1987

6. F. Reynaud, H. Lagorceix, "Stabilization and control of a fiber array for the coherent transport of beams in a stellar interferometer", Integrated Optics for Astronomical Interferometry, eds. Grenoble, France: Bastianelli-Guirimand, pp.249-257, 1997.

7. L. Delage and F. Reynaud, "Kilometric optical fiber interferometer", Optics express, 9, 6, pp. 267-271, 2001. 
8. Y. Machida, J. Nishikawa, K. Sato, T. Fukushima, M. Yoshizawa, Y. Honma, Y. Torii, K. Matsuda, K. Kubo, M. Ohashi, S. Suzuki., and H. Iwashita, "MIRA-I Project: Development of Mitaka Optical and Infrared Stellar Interferometer", Proc. of SPIE, 3350, pp. 202-211, 1998.

9. K. Sato, J. Nishikawa, M. Yoshizawa, T. Fukushima, Y. Machida, Y. Honma, R. Kuwabara, S. Suzuki., Y. Torii, K. Kubo, K. Matsuda, and H. Iwashita, "Development of the optical and infrared interferometer, MIRA-I.2", Proc. of SPIE, 3350, pp. 212-217, 1998.

10. J. Nishikawa, "Status report on Mitaka optical and InfraRed Array (MIRA-I.2)", Proc. of SPIE, 4838, submitted, 2002.

11. K. Sato, J.Nishikawa, M. Yoshizawa, T. Fukushima, Y. Torii, K. Matsuda, K. Kubo, H. Iwashita, S. Suzuki, D. Saint-Jacques, "Experiment of the fiber-connected interferometer for MIRA project", Proc. of SPIE, 4006, pp. 11029-1106, 2000.

12. F. Reynaud, J. J. Alleman, and P. Connes, "Interferometric control of fiber length for a coherent telescope array", Appl. Opt. 31, 19, pp. 3736-3743, 1992.

13. G. Perrin, O. Lai, J. Woillez, J. Guerin, A. J. Adamson, O. Guyon, P. J. Lena, J. Nishikawa, F. Reynaud, S. T. Ridgway, F. J. Rigaut, A. T. Tokunaga, P. L. Wizinowich, "OHANA: the Optical Hawaiian Array for nanoradian astronomy on top of Mauna Kea", Proc. of SPIE, 4838, submitted, 2002.

14. G. Perrin, O. Lai, J. Woillez, J. Guerin, F. Reynaud, S. T. Ridgway, P. J. Lena, P. L. Wizinowich, A. T. Tokunaga, J. Nishikawa, F. J. Rigaut, A. J. Adamson, O. Guyon, "OHANA phase II: a prototype demonstrator of fiber linked interferometry between very large telescopes", Proc. of SPIE, 4838, submitted, 2002.

15. O. Lai, S. T. Ridgway, G. Fahlman, G. Perrin, P. J. Lena, A. J. Adamson, "OHANA phase III: scientific operation of an 800-meter Mauna Kea interferometer", Proc. of SPIE, 4838, submitted, 2002.

16. S. T. Ridgway, O. Lai, G. Perrin, "Optical delay concepts for OHANA", Proc. of SPIE, 4838, submitted, 2002. 